\title{
Reviewer Acknowledgement 2019
}

\author{
Klaus A. Hausegger ${ }^{1}$
}

(C) Springer Science+Business Media, LLC, part of Springer Nature and the Cardiovascular and Interventional Radiological Society of Europe (CIRSE) 2019

In order to maintain high scientific standards for Cardiovascular and Interventional Radiology (CVIR), our Editors relay on our large pool of expert reviewers, who carefully assess manuscripts and write review comments. Peer review lies at the heart of scientific publishing and is a time-consuming and often complex task that is vital to the success of CVIR. Therefore, on behalf of all CVIR Editors, I would like to gratefully acknowledge all of our reviewers for the valuable time and effort invested in reviewing for the journal. The continuing success of CVIR would not be possible without their generous work.

The new General Data Protection Regulation (GDPR) legislation prevents us from publishing the names of reviewers as we have in the past. Nevertheless, we are indebted to the more than 500 experts who have provided in-depth reviews of manuscripts submitted for publication in CVIR within the last 12 months.

If you wish to receive a peer-review certificate, please contact the CVIR Editorial Office.

Thank you to all of our reviewers for their support and contributions in 2019. We look forward to another successful year!

Klaus A. Hausegger, MD

Editor-in-Chief CVIR

On behalf of all CVIR Editors

\section{Compliance with Ethical Standards}

Conflict of interest The author declares that he has no conflict of interest.
Klaus A. Hausegger

CVIR.Hausegger@kabeg.at

1 Department of Diagnostic and Interventional Radiology, Klagenfurt State Hospital, Klagenfurt, Austria 